\title{
Cancer therapeutic implications of microRNAs in the regulation of immune checkpoint blockade
}

\author{
Yan Zhang ${ }^{1 *}$, Toshihiko Tanno ${ }^{1}$ and Chrysi Kanellopoulou ${ }^{2}$
}

\begin{abstract}
Dampening of patients' immune response to tumor-specific antigens is a major reason for tumor development and progression. Within tumor microenvironment, aberrant expression of immune checkpoints changes T lymphocyte activity, and induces immune tolerance or escape. Inhibiting these checkpoints, called immune-checkpoint blockade, causes the re-activation of the immune response to suppress tumor progression. microRNAs (miRNAs) are small non-coding RNAs that regulate multiple biological processes including carcinogenesis. miRNAs directly and indirectly control the expression of checkpoint receptors and can benefit the immune checkpoint blockade. In this review, we summarize what is known about miRNAs that regulate the expression of various immune checkpoints in cancer therapy and discuss the clinical implications that miRNAs could be useful therapeutic partners assisting the immune checkpoint therapy.
\end{abstract}

Keywords: Immune checkpoint blockade, CTLA-4, PD-1, PD-L1, microRNA

The human immune system is an intricate cellular network, which has developed to protect us against microorganism invasion but can also eliminate malignant cells in our body. Thereby, cancer is considered as another manifestation of immune-deficiency, due to the fragile anti-tumor immunity in most patients [1]. The immune system is classified into two parts: innate immunity and adaptive immunity. Unlike innate immune responses, adaptive immunity functions against specific antigens and also leads to the development of immunological memory [2]. As a major anti-tumor executor, adaptive immunity includes multiple types of professional immune cells, such as T, B and NK cells. T cells have been extensively studied in the context of adaptive immunity; they can be activated by specific antigen, and then precisely recognize and kill the host cells which bear those antigens [2]. Interestingly, after $\mathrm{T}$ cells are activated, they always up-regulate a group of cell surface proteins, called inhibitory immune checkpoint proteins, which can shut off the ongoing immune response and suppress

\footnotetext{
* Correspondence: Yan.Zhang@ihv.umaryland.edu

${ }^{1}$ Division of Immunotherapy, Institute of Human Virology (IHV), School of

Medicine, University of Maryland, Baltimore, USA

Full list of author information is available at the end of the article
}

$\mathrm{T}$ cell function [3]. The major inhibitory receptors on $\mathrm{T}$ cells include PD-1, CTLA-4, LAG-3, TIM-3, and BTLA, of which the best characterized are CTLA4 and PD-1 [3]. CTLA4 and PD-1 are both up-regulated in activated T cells. CTLA4 is also highly expressed on a special type of CD4 T cells, regulatory T cells (Tregs). CTLA4 can bind to CD80/CD86, which is highly expressed on antigen presenting cells (APCs). After binding, activated T cells will receive a negative signal from this immune checkpoint, and mitigate the $\mathrm{T}$ cell mediated immune response. PD-1 can bind to PD-L1 or PD-L2 on the APC cells and induce $\mathrm{T}$ cell apoptosis and thus suppress the $\mathrm{T}$ cell immune response. Since an unleashed immune response would cause inflammation and autoimmunity in human beings, inhibitory immune checkpoints, which are the products of millenia of evolution, serve as a balance for proper immune response [3, 4].

In the tumor microenvironment, some tumor cells hijack this negative feed-back regulation loop by enhancing the suppression activity of inhibitory immune checkpoints, and thus escape immune surveillance of malignant cells $[4,5]$. Tumor cells often express novel antigens, termed neo-antigens, which are not expressed in normal cells and thus are not recognized as 'self'.

(c) The Author(s). 2019 Open Access This article is distributed under the terms of the Creative Commons Attribution 4.0 International License (http://creativecommons.org/licenses/by/4.0/), which permits unrestricted use, distribution, and reproduction in any medium, provided you give appropriate credit to the original author(s) and the source, provide a link to the Creative Commons license, and indicate if changes were made. The Creative Commons Public Domain Dedication waiver (http://creativecommons.org/publicdomain/zero/1.0/) applies to the data made available in this article, unless otherwise stated. 
When $\mathrm{T}$ cells detect these neo-antigens on tumor cells, they can efficiently remove them. However, most tumor cells highly express the ligands, which can bind to the inhibitory immune checkpoint proteins on $\mathrm{T}$ cells, and induce immune suppression. For example, PD-L1 is a specific ligand for PD-1, which transmits inhibitory signaling to $\mathrm{T}$ cells and reduces $\mathrm{T}$ cell proliferation and cell viability. PD-L1 is normally expressed on antigen presenting cells, such as macrophages and dendritic cells and plays an important role in multiple physiological events, such as restricting $\mathrm{T}$ cell responses after viral clearance. However, in some tumor cells, PD-L1 expression level is aberrantly elevated which induces tolerance of tumor infiltrating T cells. Besides PD-L1, CEACAM1 is another inhibitory immune checkpoint, which is also expressed on tumor cells [6].

Recently, the application of immune checkpoint blockade in tumor immuno-therapy led to a big breakthrough of the management of late stage cancers. By targeting the inhibitory immune checkpoint proteins on immune cells with antibodies, the immune system could be re-activated to suppress tumor progression $[4,5,7]$. This greatly improves the prognosis in multiple human cancer patients.

Two distinct inhibitory immunological checkpoint blockades, CTLA-4: CD80/CD86 interactions and PD-1: PD-L1 interactions, are broadly used in anti-tumor immunotherapy. Ipilimumab, was the first commercial checkpoint drug approved by FDA for cancer immunotherapy at $2011[8,9]$. It targets the CTLA4 protein on $\mathrm{T}$ cells and inhibits $\mathrm{T}$ cell repression thus enhancing the immune response against tumor cells. Nivolumab, which is a commercial anti-PD-1 mAb, blocks the binding between PD-1 and PD-L1, and thereby help T cells escape from immune suppression in cancer patients. Both antibodies show exciting results in patients. Although this new therapy is approved for numerous types of cancer, only part of patients can benefit from it. Introduction of other genetic or epigenetic markers could further expand the application of the immune checkpoint blockade in the clinical cancer therapy [10].

The human genome only contains about 20,000 protein genes, whose sequences account for $\sim 1.5 \%$ of the genome. The rest of the human genome is comprised primarily of non-coding RNAs and repetitive elements. A lot of interest in non-coding RNAs originated by the discovery of a group of small non-coding RNAs, named microRNAs (miRNAs) [11]. miRNAs are small non-coding RNAs, around 22 nt in length [12]. Although originally described in $C$. elegans they are highly conserved in many species, including Drosophila and humans. miRNA genes are transcribed into long primary microRNA (pri-miRNA) in the nucleus, usually by polymerase II (polII). They are then sequentially processed by two RNAseIII enzymes, Drosha in the nucleus, which releases a $40-60 \mathrm{nt}$ pre-miRNA from the pri-miRNA, and Dicer which further cleaves to generate a mature 22 nt miRNA. miRNAs exert post-transcriptional control of protein expression by binding to the 3 '-arm untranslated region $\left(3^{\prime}-\mathrm{UTR}\right)$ of target genes'. When the miRNA is fully complementary to the target gene mRNA, it leads to cleavage of transcripts, but there are few examples of this in vivo [13]. Usually miRNAs bind with imperfect complementarity their cognate targets and induce RNA de-adenylation and protein translation inhibition [14-16]. There are about 2000 miRNA genes have been annotated in the human genome but the recent data indicate that the real number is closer to 600 [16]. Each miRNA normally can regulate multiple genes expression, and meanwhile each gene transcript can also be targeted by several different miRNAs. Complete ablation of miRNAs in animal models by deletion of Dicer or Drosha is embryonic lethal and miRNAs are involved in almost every critical cellular processes during development $[17,18]$. Dysregulation or mutation of miRNAs is also associated with various human diseases, including cancer $[18,19]$. miRNAs can also be secreted by cells and circulate in human blood or other body fluids. These circulating miRNAs are not only good disease diagnostic biomarkers, but can also be absorbed by tissue cells and regulate target gene expression in a paracrine manner [20]. However, the function of miRNAs on immunocheckpoint during the pathogenesis of cancer is rarely reported. Here we summarized the recent progress and discuss the application potential of miRNA in immunocheckpoint related immuno-therapy.

\section{microRNAs regulate the expression of immune checkpoint molecules}

Recent studies demonstrated that miRNAs regulate the expression of different immune checkpoints; these include the checkpoint molecules expressed on $\mathrm{T}$ cells, like PD-1, CTLA-4, LAG-3 and BTLA, but also the ones on tumor cells and APC cells, like PD-L1, CEACAM1, B7-H3 and CD80. One miRNA can target several checkpoints and mimics the effect of combination therapy.

\section{Checkpoints in $\mathrm{T}$ cells}

As mentioned above, in $\mathrm{T}$ cells, there are many inhibitory immune checkpoints. PD- 1 and CTLA- 4 are the best characterized ones and have proven to be effective targets for immunotherapy against cancer. It's reported in a mouse Glioma model, miRNA-138 bind the 3 '-UTR region of both Ctla4 and Pd-1 genes and inhibit both PD-1 and CTLA4 protein expression. Hence miRNA-138 can enhance the mouse immune response and attenuate tumor progression [21]. Besides miRNA-138, miRNA-28 is also shown to inhibit PD-1 expression in T cells [22]. 
Low levels of miRNA-28 cause $\mathrm{T}$ cell exhaustion, and allow tumor cells to escape immune surveillance in a mouse melanoma model. Another two important immune checkpoint proteins on T cells are TIM-3 and BTLA. T-cell immunoglobulin and mucin-domain containing-3 (TIM-3) is present on activated $\mathrm{T}$ effector cells and negatively controls the responses of $\mathrm{T}$ effector cells by inducing $\mathrm{T}$ cell tolerance and exhaustion. BTLA binds HVEM (Herpesvirus Entry Mediator) and suppresses the CD8 T cell mediated immune response. BTLA expression is down regulated during $\mathrm{T}$ cell differentiation, but it's also noted that tumor-infiltrating CD8+ $\mathrm{T}$ cells express high levels of BTLA [23], which then accelerates tumor progression. microRNA-28 can target both BTLA and TIM-3 genes, and down regulates their expression [24].

\section{Checkpoints in tumor cells}

Although tumor cells are not professional antigen presenting cells and have lower affinity to $\mathrm{T}$ cells, they do express specific immune checkpoint proteins on their cell surface. The two major immune checkpoint proteins on tumor cells are PD-L1 and CEACAM1. In 3'UTR region of PD-L1 gene, there are multiple miRNA binding sites. Meanwhile, tumor cells express more miRNAs, maybe due to aberrant transcription regulation [25]. Thereby, miRNAs regulation of PD-L1 expression and subsequent immune responses is frequently reported in different types of cancer. For example, in a gastric cancer tissue sample, a single nucleotide mutation on the 3'-UTR of the PD-L1 gene, which disrupted the miR-570 binding was reported. This mutation correlated with enhanced PD-L1 expression and a more aggressive cancer phenotype [26]. By now many miRNAs which can bind the 3'-UTR of the PD-L1 gene and can down-regulate the PD-L1 expression have been described. These miRNAs include: miR-570, 34a, 200, 21 and 197 [26-30]. Although all these miRNAs can down-regulate PD-L1 and expose tumor cells to T cell mediated killing, their expression level is pretty low in aggressive tumor cells. On the other hand, p53, which is a well-known anti-tumor effector protein can directly enhance the miR-34a expression, and block the PD-L1 mediated inhibitory immune checkpoint, which might contribute to the tumor suppressor properties of p53 [27]. These findings indicate that PD-L1 might be a major target for miRNA control of immune checkpoints.

B7-H3 is another important immune checkpoint, which is expressed on many different types of cells, including some solid tumors. Although the function of B7-H3 is still controversial, miRNA-29c, which can down-regulate $\mathrm{B} 7-\mathrm{H} 3$ protein expression shows good correlation with better prognosis in breast cancer patients. This hints that B7-H3 is potentially a valid inhibitory immune checkpoint molecule and can be used as a good target for miRNA mediated anti-tumor immune response [31, 32].

\section{Checkpoints in APCs}

APC cells are professional immune cells interacting with $\mathrm{T}$ cells and modulating $\mathrm{T}$ cell immune responses. They are normally express a number of proteins involved in immune checkpoints, such as PD-L1, B7-H3, CD80, and CD86 to dampen the immune response once antigen is cleared. Since we have discussed miRNA regulation of PD-L1 and B7-H3 in tumor cells, here we focus on CD80. CD80 is not expressed on tumor cells, but it still can transmit an inhibitory signal to $\mathrm{T}$ cells, by interacting with CTLA4. In APC cells, such as dendritic cells, overexpression of miRNA-424 (322) can reduce CD80 protein expression and thereby block the CTLA4/CD80 mediated inhibitory immune checkpoint pathway [33].

All these data indicate that miRNAs could function similar as some checkpoint antibodies and regulate the anti-tumor $\mathrm{T}$ cell immune response by effecting on the expression of different checkpoint molecules.

\section{Two microRNAs predict the response of immune checkpoint blockade}

Clinical data have shown that only around $20 \%$ of the patients respond to immune checkpoint related immuno-therapy [34]. Therefore, it would be important to know in advance who would benefit from the immune checkpoint blockade therapy.

miRNAs have been excellent biomarkers for a number of diseases [35]. Therefore, a reasonable strategy to predict if a patient will respond to checkpoint inhibitors could be the evaluation of miRNAs that control the level of immune checkpoints. One possible biomarker for selecting patients who would benefit from anti-PD-L1 therapy is miR-34a. By using bone marrow samples from 44 acute myeloid leukemia and 5 healthy controls, the researchers reported that the level of miR-34a is inversely correlated with that of PD-L1, suggesting that patients with low miR-34a levels might benefit more from PD-L1 blockade [36]. In addition, in mesothelioma tumor samples, high levels of PD-L1 correlate with a low level of miR-15b, miR-16, miR-193a-3p and miR-200c, which predict a poor prognosis. Another study found that the miR-200 family correlates inversely with the mRNA level of PD-L1 by using the TCGA lung adenocarcinoma database $(n=230)$, indicating that the low level of miR-200 is a suitable biomarker to predict lung adenocarcinomas, which will respond well to immune checkpoint blockade [29]. All these studies suggest that microRNAs are useful in predicting therapeutic efficacy of immune checkpoint blockade. 
Further research is necessary to discover new biomarkers specific for the molecular mechanism of immune checkpoint inhibition.

\section{3 miRNAs intensify the immune checkpoint blockade efficacy}

As previously mentioned, only a subset of patient benefits from immune checkpoint blockade therapy. Combinations of immune checkpoint inhibitors increase the therapeutic efficacy. Since miRNAs who can target multiple immune checkpoints simultaneously they may be a good choice to mimic combination therapy of immune checkpoint blockade.

Combination with the anti-PD-1 mAb, Nivolumab, and anti-CTLA-4 mAb Ipilimumab, which significantly increased patients' response rates of advanced melanoma patients $[7,8]$, is the most frequently used combination in cancer therapy. It has been show that miR-138 is able to target both PD-1 and CTLA4, suggesting its potential to mimic the combined therapeutic effect of anti-PD-1 and anti-CTLA-4. miRNA-138 binds to the 3'UTR of PD- 1 and CTLA-4 and down-regulates the expression of these checkpoints in vitro and in vivo. miRNA-138 treatment on activated the T-cells increased the survival rate of immune-competent glioma mice by $43 \%$. As expected, miRNA-138 did not change the survival rate in immune-compromised mice, indicating that miRNA-138 functions mainly through modulating the mouse immune system [21].

Some other miRNAs, which can target multiple immune checkpoints, have been discovered as well. One is miRNA-28; in vitro studies demonstrated that miRNA-28 mimics are able to decrease the expression of PD-1, while miRNA-28 inhibition leads to increased PD-1, TIM3 and BTLA35 [22]. Another one is miR-424. It was shown that, in ovarian cancer tumors, the expression of miR-424 is negatively associated with the level of PD-L1 and CTLA-4 ligand CD80. High level of miR-424 is correlated with progression free survival. Restoration of miR-424 levels in a tumor model led to $\mathrm{T}$ cell activation and reversed chemo-resistance. Therefore, introducing miRNA-424 mimetics to the immune checkpoint blockade can increase the therapeutic efficiency of immunotherapy [33].

On the other hand, combination of miRNAs that targeting on different immune checkpoints, for example, mixture of miRNA-28 and miRNA-424 mimics to inhibit PD-1 and CTLA-4 simultaneously, may mimic the therapeutic effect improvement of a combined immune checkpoint blockade. This type of combination needs to be confirmed by more in vivo and clinical studies.

\section{Conclusions and discussion}

Inhibition of immune checkpoints with neutralizing antibodies has demonstrated promise in a variety of malignancies. However, it is still relatively restricted to specific tumor types and stages. Because miRNAs regulate the transcription of immune checkpoint, adding miRNAs in cancer immune therapy regimens could lay the foundation for a very exciting and innovative approach to increase the potency of immune checkpoint blockade. First of all, defined miRNA patterns are associated with specific alterations in immune checkpoint pathways in the tumor microenvironment, which may provide a accurate biomarker predicting patients response to immune checkpoint inhibition. Also, since one miRNA can target multiple immune checkpoint molecules, miRNAs can augment the immune checkpoint blockade efficacy by mimicking combination therapy. In addition, combination of miRNAs with different immune checkpoint targets may also mimic the effect of a combined immune checkpoint blockade therapy.

Meanwhile, other types of non-coding RNAs may also be involved in functional modulation of immune checkpoints. For example, secreted miRNAs in plasma can be used as biomarkers for patients' response to cancer therapy [37]. In the tumor microenvironment, it is important to find out if the microRNAs that control the expression of immune checkpoints can be secreted by exosomes to effect on other cells. Some longer noncoding RNAs [38, 39] have been predicted to regulate immune checkpoints as well. Recently one study has reported that the level of the lncRNA actin filament-associated protein 1 antisense RNA1 (AFAP1-AS1) is positively correlated with that of PD1 in nasopharyngeal cancer tissues [40]. Therefore, study of the role of IncRNAs and other types of ncRNAs in the regulation of immune checkpoint blockade is necessary to complete the regulatory network of immune checkpoints.

In conclusion, miRNAs are key regulatory elements of tumor immune evasion by regulating immune checkpoints. As has been described for other biological processes a number of miRNAS build an intricate network that partially controls the immune response by targeting key immune checkpoint proteins. This opens up the possibility of using them as a promising therapeutic in cancer immune therapy regimens.

\footnotetext{
Abbreviations

3'-UTR: 3'-arm untranslated region; AFAP1-AS1: actin filament-associated protein 1 antisense RNA 1; APCs: Antigen presenting cells; BTLA: B- and T-

lymphocyte attenuator; CTLA-4: Cytotoxic T-lymphocyte-associated protein 4; HVEM: Herpesvirus Entry Mediator; LAG-3: Lymphocyte-activation gene 3: miRNA: microRNA; PD-1: Programmed cell death protein 1; PD-

L1: Programmed death-ligand 1; pri-miRNA: primary microRNA; TIM-3: T cell immunoglobulin and mucin-domain containing-3; Tregs: regulatory T cells
}

Acknowledgements

Not applicable. 


\section{Funding}

Not applicable.

\section{Availability of data and materials} Not applicable.

\section{Authors' contributions}

Y.Z. and C.K. wrote the manuscript. T.T. and C.K. modified the manuscript. All authors read and approved the final manuscript.

\section{Ethics approval and consent to participate}

Not applicable.

\section{Consent for publication}

All the authors agree to the publication.

\section{Competing interests}

The authors declare that they have no competing interests.

\section{Publisher's Note}

Springer Nature remains neutral with regard to jurisdictional claims in published maps and institutional affiliations.

\section{Author details}

${ }^{1}$ Division of Immunotherapy, Institute of Human Virology (IHV), School of Medicine, University of Maryland, Baltimore, USA. ${ }^{2}$ Laboratory of Immunology, NIAID, National Institutes of Health, Bethesda, Maryland, USA.

Received: 1 August 2018 Accepted: 14 March 2019

Published online: 02 August 2019

\section{References}

1. Qu X, Tang Y, Hua S. Immunological approaches towards Cancer and inflammation: a cross talk. Front Immunol. 2018;9:563.

2. Medzhitov R. Recognition of microorganisms and activation of the immune response. Nature. 2007:449(7164):819-26.

3. Wherry EJ, Kurachi M. Molecular and cellular insights into T cell exhaustion. Nat Rev Immunol. 2015;15(8):486-99.

4. Joyce JA, Fearon DT. T cell exclusion, immune privilege, and the tumor microenvironment. Science. 2015;348(6230):74-80.

5. Liebelt BD, Finocchiaro G, Heimberger AB. Principles of immunotherapy. Handb Clin Neurol. 2016;134:163-81.

6. Dankner M, Gray-Owen SD, Huang YH, Blumberg RS, Beauchemin N CEACAM1 as a multi-purpose target for cancer immunotherapy. Oncoimmunology. 2017;6(7):e1328336.

7. Topalian SL, Drake CG, Pardoll DM. Immune checkpoint blockade: a common denominator approach to cancer therapy. Cancer Cell. 2015:27(4):450-61.

8. Mokyr MB, Kalinichenko T, Gorelik L, Bluestone JA. Realization of the therapeutic potential of CTLA-4 blockade in low-dose chemotherapytreated tumor-bearing mice. Cancer Res. 1998;58:5301-4.

9. Hodi FS, O'Day SJ, McDermott DF, et al. Improved survival with ipilimumab in patients with metastatic melanoma. N Engl J Med. 2010:363:711-23.

10. Sharma P, Allison JP. The future of immune checkpoint therapy. Science 2015 Apr 3;348(6230):56-61.

11. Wightman $\mathrm{B}, \mathrm{Ha}$ I, Ruvkun G. Posttranscriptional regulation of the heterochronic gene lin-14 by lin-4 mediates temporal pattern formation in C. elegans, Cell. 1993;75(5):855-62.

12. Ambros $\vee$. The functions of animal microRNAs. Nature. 2004 Sep 16: 431(7006):350-5.

13. Yekta S, Shih $\mathrm{H}$, Bartel DP. MicroRNA-directed cleavage of HOXB8 mRNA. Science. 2004;304(5670):594-6.

14. Giraldez AJ, Mishima Y, Rihel J, Grocock RJ, Van Dongen S, Inoue K, Enright AJ, Schier AF. Zebrafish MiR-430 promotes deadenylation and clearance of maternal mRNAs. Science. 2006;312(5770):75-9.

15. Bartel DP. MicroRNAs: genomics, biogenesis, mechanism, and function. Cell. 2004 Jan 23;116(2):281-97.

16. Fromm B, Billipp T, Peck LE, Johansen M, Tarver JE, King BL, Newcomb JM, Sempere LF, Flatmark K, Hovig E, Peterson KJ. A uniform system for the annotation of vertebrate microRNA genes and the evolution of the human microRNAome. Annu Rev Genet. 2015;49:213-42.
17. Bartel DP. Metazoan MicroRNAs. Cell. 2018;173(1):20-51.

18. Jiang $Q$, Wang $Y$, Hao $Y$, Juan $L$, Teng M, Zhang X, Li M, Wang G, Liu Y. miR2Disease: a manually curated database for microRNA deregulation in human disease. Nucleic Acids Res. 2009:37(Database issue):D98-104.

19. Calin GA, Dumitru CD, Shimizu M, Bichi R, Zupo S, Noch E, Aldler H, Rattan S, Keating M, Rai K, Rassenti L, Kipps T, Negrini M, Bullrich F, Croce CM. Frequent deletions and down-regulation of micro- RNA genes miR15 and miR16 at 13q14 in chronic lymphocytic leukemia. Proc Natl Acad Sci U S A. 2002;99(24):15524-9.

20. Chen X, Ba Y, Ma L, Cai X, Yin Y, Wang K, Guo J, Zhang Y, Chen J, Guo X, Li Q, Li X, Wang W, Zhang Y, Wang J, Jiang X, Xiang Y, Xu C, Zheng P, Zhang J, Li R, Zhang H, Shang X, Gong T, Ning G, Wang J, Zen K, Zhang J, Zhang CY. Characterization of microRNAs in serum: a novel class of biomarkers for diagnosis of cancer and other diseases. Cell Res. 2008;18(10):997-1006.

21. Wei J, Nduom EK, Kong LY, Hashimoto Y, Xu S, Gabrusiewicz K, Ling X, Huang N, Qiao W, Zhou S, Ivan C, Fuller GN, Gilbert MR, Overwijk W, Calin GA, Heimberger AB. MiR-138 exerts anti-glioma efficacy by targeting immune checkpoints. Neuro-Oncology. 2016;18(5):639-48.

22. Li Q, Johnston N, Zheng X, Wang H, Zhang X, Gao D, Min W. miR-28 modulates exhaustive differentiation of $T$ cells through silencing programmed cell death-1 and regulating cytokine secretion. Oncotarget. 2016;7(33):53735-50

23. Paulos $\mathrm{CM}$, June $\mathrm{CH}$. Putting the brakes on BTLA in T cell-mediated cancer immunotherapy. J Clin Invest. 2010;120(1):76-80.

24. Das M, Zhu C, Kuchroo VK. Tim-3 and its role in regulating anti-tumor immunity. Immunol Rev. 2017 Mar;276(1):97-111.

25. Adams BD, Kasinski AL, Slack FJ. Aberrant regulation and function of microRNAs in cancer. Curr Biol. 2014;24(16):R762-76. https://doi.org/10.1016/ j.cub.2014.06.043.

26. Wang W, Sun J, Li F, Li R, Gu Y, Liu C, Yang P, Zhu M, Chen L, Tian W, Zhou H, Mao Y, Zhang L, Jiang J, Wu C, Hua D, Chen W, Lu B, Ju J, Zhang X. A frequent somatic mutation in CD274 3'-UTR leads to protein overexpression in gastric cancer by disrupting miR-570 binding. Hum Mutat. 2012;33(3):480-4.

27. Cortez MA, Ivan C, Valdecanas D, Wang X, Peltier HJ, Ye Y, Araujo L, Carbone DP, Shilo K, Giri DK, Kelnar K, Martin D, Komaki R, Gomez DR, Krishnan S, Calin GA, Bader AG, Welsh JW. PDL1 regulation by $p 53$ via miR-34. J Natl Cancer Inst. 2015:17:108(1).

28. Fujita Y, Yagishita S, Hagiwara K, Yoshioka Y, Kosaka N, Takeshita F, Fujiwara T, Tsuta K, Nokihara H, Tamura T, Asamura H, Kawaishi M, Kuwano K, Ochiya T. The clinical relevance of the miR-197/CKS1B/STAT3-mediated PD-L1 network in chemoresistant non-small-cell lung cancer. Mol Ther. 2015:23(4):717-27

29. Chen L, Gibbons DL, Goswami S, Cortez MA, Ahn YH, Byers LA, Zhang X, Yi $X$, Dwyer D, Lin W, Diao L, Wang J, Roybal J, Patel M, Ungewiss C, Peng D, Antonia S, Mediavilla-Varela M, Robertson G, Suraokar M, Welsh JW, Erez B, Wistuba II, Chen L, Peng D, Wang S, Ullrich SE, Heymach JV, Kurie JM, Qin FX. Metastasis is regulated via microRNA-200/ZEB1 axis control of tumour cell PD-L1 expression and intratumoral immunosuppression. Nat Commun. 2014:5:5241

30. Zhu J, Chen L, Zou L, Yang P, Wu R, Mao Y, Zhou H, Li R, Wang K, Wang W, Hua D, Zhang X. MiR-20b, -21, and -130b inhibit PTEN expression resulting in B7-H1 over-expression in advanced colorectal cancer. Hum Immunol. 2014;75(4):348-53.

31. Picarda E, Ohaegbulam KC, Zang X. Molecular pathways: targeting B7-H3 (CD276) for human Cancer immunotherapy. Clin Cancer Res. 2016;22(14):3425-31.

32. Nygren MK, Tekle C, Ingebrigtsen VA, Mäkelä R, Krohn M, Aure MR, NunesXavier CE, Perälä M, Tramm T, Alsner J, Overgaard J, Nesland JM, Borgen E, Børresen-Dale AL, Fodstad $\varnothing$, Sahlberg KK, Leivonen SK. Identifying microRNAs regulating B7-H3 in breast cancer: the clinical impact of microRNA-29c. Br J Cancer. 2014;110(8):2072-80.

33. Xu S, Tao Z, Hai B, Liang H, Shi Y, Wang T, Song W, Chen Y, OuYang J, Chen J, Kong F, Dong Y, Jiang SW, Li W, Wang P, Yuan Z, Wan X, Wang C, Li W, Zhang X, Chen K. miR-424(322) reverses chemoresistance via T-cell immune response activation by blocking the PD-L1 immune checkpoint Nat Commun. 2016;7:11406.

34. Schadendorf D, Hodi FS, Robert C, Weber JS, Margolin K, Hamid O, Patt D, Chen T, Berman DM, Wolchok JD. Pooled analysis of Long-term survival data from phase II and phase III trials of Ipilimumab in Unresectable or metastatic melanoma. J Clin Oncol. 2015:33(17):1889-94. 
35. Kim YK. Extracellular microRNAs as biomarkers in human disease. Chonnam Med J. 2015;51(2):51-7. https://doi.org/10.4068/cmj.2015.51.2.51 Epub 2015 Aug 17.

36. Wang $X$, Li J, Dong $K$, Lin F, Long $M$, Ouyang $Y$, Wei J, Chen X, Weng Y, He T, Zhang H. Tumor suppressor miR-34a targets PD-L1 and functions as a potential immunotherapeutic target in acute myeloid leukemia. Cell Signal. 2015;27(3):443-52.

37. Braicu C, Tomuleasa C, Monroig P, Cucuianu A, Berindan-Neagoe I, Calin GA. Exosomes as divine messengers: are they the Hermes of modern molecular oncology? Cell Death Differ. 2015;22(1):34-45.

38. Bullock MD, Silva AM, Kanlikilicer-Unaldi P, Filant J, Rashed MH, Sood AK, Lopez-Berestein G, Calin GA. Exosomal non-coding RNAs: diagnostic, prognostic and therapeutic applications in Cancer. Noncoding RNA. 2015;1(1):53-68.

39. Bullrich F, Fujii H, Calin G, Mabuchi H, Negrini M, Pekarsky Y, Rassenti L, Alder H, Reed JC, Keating MJ, Kipps TJ, Croce CM. Characterization of the $13 q 14$ tumor suppressor locus in CLL: identification of ALT1, an alternative splice variant of the LEU2 gene. Cancer Res. 2001;61(18):6640-8.

40. Tang YY, He Y, Shi L, Yang LT, Wang JP, Lian Y, et al. Coexpression of AFAP1-AS1 and PD-1 predicts poor prognosis in nasopharyngeal carcinoma. Oncotarget. 2017;8:39001-11.

Ready to submit your research? Choose BMC and benefit from:

- fast, convenient online submission

- thorough peer review by experienced researchers in your field

- rapid publication on acceptance

- support for research data, including large and complex data types

- gold Open Access which fosters wider collaboration and increased citations

- maximum visibility for your research: over $100 \mathrm{M}$ website views per year

At $\mathrm{BMC}$, research is always in progress.

Learn more biomedcentral.com/submissions 